\title{
Perioperative single photon emission computed tomography in predicting survival of malignant glioma patients
}

\author{
VYTENIS DELTUVA ${ }^{1}$, ADOMAS BUNEVICIUS ${ }^{1}$, NEMIRA JURKIENE ${ }^{2}$, \\ ILONA KULAKIENE $^{2}$ and ARIMANTAS TAMASAUSKAS ${ }^{1}$ \\ Departments of ${ }^{1}$ Neurosurgery and ${ }^{2}$ Nuclear Medicine, \\ Hospital of the Lithuanian University of Health Sciences, Kaunas, Lithuania
}

Received March 23, 2012; Accepted June 25, 2012

DOI: $10.3892 / 01.2012 .812$

\begin{abstract}
Single photon emission computed tomography (SPECT) is widely used in the evaluation of glioma patients and has been demonstrated to correlate with glioma malignancy and proliferation indexes. The aim of this study was to evaluate the association between perioperative technetium-99m-methoxyisobutylisonitrile ( ${ }^{99} \mathrm{~m}$ Tc-MIBI) uptake on SPECT scans and survival of malignant glioma patients. A total of 17 patients (11 males and 6 women; mean age, 62.2 \pm 8.4 years) with histologically confirmed malignant gliomas (16 glioblastoma multiforme and 1 gliosarcoma) underwent ${ }^{99 \mathrm{~m}} \mathrm{Tc}-\mathrm{MIBI}$ SPECT scans $2.8 \pm 1.9$ days before surgery and $9.8 \pm 1.5$ days after surgery. The total intensity index (TII) that corresponds to the area and intensity of tracer uptake was calculated before and after surgery. In addition, the change of TII before versus after surgery ( $\Delta$ TII) was calculated. The overall survival (OS) was defined as the period between the date of surgery and the date of death. The median overall survival time was 12.4 months, ranging from 1.4 to 88 months; there were nine (45\%) 12-month survivors. In univariate analyses using a log-rank test, worse OS was significantly associated with higher preoperative TII $(\geq 12)$, higher postoperative TII $(\geq 6)$, lower $\Delta$ TII $(<50 \%)$ and higher number of neurological symptoms prior to surgery $(\geq 4)$. In multivariate analyses, higher postoperative TII, a greater number of neurological symptoms and female gender were found to be factors with independent prognostic value of OS. Patients who survived more than 12 months following surgery had a significantly lower postoperative TII, higher $\Delta$ TII and greater rate of gross total resection compared to patients who survived less than 12 months following surgery. Higher perioperative tracer uptake and lower decrease of tracer uptake following surgery (suggesting less radical resection) were associated with worse OS of malignant glioma patients. Our
\end{abstract}

Correspondence to: $\mathrm{Dr}$ Adomas Bunevicius, Department of Neurosurgery, Hospital of Lithuanian University of Health Sciences, Eiveniu g. 2, LT-50009, Kaunas, Lithuania

E-mail: a.bunevicius@yahoo.com

Key words: glioma, single photon emission tomography, survival results suggest that SPECT may be used to predict survival of malignant glioma patients; however, further studies using larger samples are required.

\section{Introduction}

Glioblastoma multiforme (GBM) is the most common malignant glioma in adults $(1,2)$. Despite recent advancements in treatment modalities that currently consist of maximally safe surgical resection followed by radiation therapy plus concomitant and adjuvant temozolomide, GBM remains an incurable and devastating disease with the median survival time rarely exceeding 14 months following diagnosis and with less than $5 \%$ of GBM patients surviving more than 3 years following diagnosis (3-5). Gliosarcoma is a rare variant of malignant glioma that is very similar to GBM in terms of genetic changes, clinical presentation and poor prognosis $(6,7)$.

Precise and timely determination of individual prognosis of malignant glioma patients is critical. To date, a number of clinical, therapeutic and genetic prognostic markers of malignant glioma patients have been identified. For example, in GBM patients, $\mathrm{O}^{6}$-methylguanine methyltransferase (MGMT) promoter methylation, good initial functional status, more radical resection and treatment with radiation therapy and chemotherapy have been demonstrated to be associated with better prognosis; while more advanced age and worse initial neurological status have been revealed to be associated with poor prognosis $(8,9)$. In addition, a new generation of genetic and biological prognostic markers is emerging, although these are not routinely used in clinical practice $(3,8,10)$. Therefore, there remains a requirement for new, reliable and widely available prognostic markers of malignant glioma.

Functional imaging methods, including positron emission tomography (PET) and single photon emission computed tomography (SPECT) play an important role in the initial diagnosis of gliomas and in the follow-up of malignant glioma patients following surgical treatment or radiotherapy when surrounding edema and gliosis make it difficult to identify remaining glioma tissue using only magnetic resonance imaging (MRI). In contrast to PET, which remains a highly expensive diagnostic modality, SPECT is widely available and routinely used, even in less developed countries. In previous studies, higher tracer uptake by glioma on SPECT was shown 
to be associated with higher grade and decreased response to chemotherapy of gliomas (11-14). In addition, higher technetium-99m-methoxyisobutylisonitrile ( ${ }^{99 \mathrm{~m} T c-M I B I) ~ u p t a k e ~ w a s ~}$ demonstrated to correlate with higher proliferative potential of gliomas and with worse survival (15-19). However, to the best of our knowledge, previous studies employed SPECT scans only once during the treatment period and currently there are no studies evaluating the association of repeated SPECT scans during perioperative periods with the prognosis of malignant glioma patients. We have recently demonstrated that changes in the bulk of malignant gliomas following surgery and radiation therapy can be reliably evaluated using SPECT (20). Changes in the bulk of malignant glioma tissue following surgery evaluated by SPECT may possibly serve as a novel prognostic marker of malignant glioma.

Therefore, in this study we aimed to evaluate the association of overall survival in malignant glioma patients with uptake of ${ }^{99 \mathrm{~m}} \mathrm{Tc}-\mathrm{MIBI}$ before and after surgery along with changes in ${ }^{99 \mathrm{~m}} \mathrm{Tc}-\mathrm{MIBI}$ uptake after surgery.

\section{Patients and methods}

Patients and protocol. Seventeen patients (11 males and 6 females; aged 62.2 \pm 8.4 years; range, 39 to 75 years) who underwent resection or biopsy for histologically confirmed grade IV glioma according to WHO classification the at the Department of Neurosurgery, Clinic of Lithuanian University of Health Sciences, Kaunas, Lithuania, were prospectively included in this study. GBM was diagnosed in $16(94 \%)$ patients and gliosarcoma was diagnosed in $1(6 \%)$ patient.

All patients underwent preoperative ${ }^{99 \mathrm{~m}} \mathrm{Tc}-\mathrm{MIBI}$ SPECT scans $2.8 \pm 1.9$ days prior to surgery or biopsy (range, 1 to 7 days). Clinical severity of the disease was evaluated by assessing the number of neurological symptoms prior to surgery or biopsy. Resection was described by a neurosurgeon as gross total (more than $90 \%$ of microscopically viable glioma removed) in $11(65 \%)$ patients or as subtotal (less than $90 \%$ of microscopically viable glioma removed) in 5 (29\%) patients. Biopsy was performed in one $(9 \%)$ patient. A postoperative ${ }^{99 \mathrm{~m}} \mathrm{Tc}-\mathrm{MIBI}$ SPECT scan was performed $9.8 \pm 1.5$ days after surgery or biopsy (range, 7 to 12 days). Following surgery, all patients received standard external beam radiation treatment that consisted of a total dose of 60 Gy that was administered in 30 fractions of 2 Gy. None of the patients received chemotherapy with temozolomide since at the time of the study temozolomide was not available in Lithuania. Survival data were obtained from the Residents' Register Service of Lithuania. Overall survival (OS) following surgery was defined as the period between the date of surgery and the date of mortality.

The study and its consent procedures were approved by the Ethics Committee of the Lithuanian University of Health Sciences, Kaunas, Lithuania, and are in agreement with Helsinki Declaration standards as well as the International Conference on Harmonization - Good Clinical Practice. All patients provided signed written informed consent.

${ }^{99 m}$ Tc-MIBI SPECT data acquisition. Radiopharmaceutical ${ }^{99 \mathrm{~m}} \mathrm{Tc}-\mathrm{MIBI}$ was used in all cases. ${ }^{99 \mathrm{~m}} \mathrm{Tc}-\mathrm{MIBI}$ is routinely used at our clinic for the evaluation of glioma patients since it is intensively uptaken by highly mitotic tumors, including high-grade, but not low-grade gliomas, normal brain tissue, necrotic tissue and fibrotic tissue $(21,22)$. During image acquisition, patients were placed in a supine position with an appropriate headpiece to avoid head movement and detectors placed as close as possible to the patients' heads. Images were captured 30 to $45 \mathrm{~min}$ after intravenous (i.v.) injection of $500 \mathrm{MBq}$ of ${ }^{99 \mathrm{~m}} \mathrm{Tc}-\mathrm{MIBI}$ using a Siemens, E. Cam dualheaded gamma camera (Siemens, Malvern, PA, USA). The matrix was set at $64 \times 64$ pixels due to the relatively small doses of ${ }^{99 \mathrm{~m}} \mathrm{Tc}-\mathrm{MIBI}$ used in the study as patients were exposed to repeated SPECT scans. The tomographic imaging parameters consisted of a $360^{\circ}$ rotation angle and an acquisition time of $30 \mathrm{sec}$ per frame with a zoom factor of 1.78. For image reconstruction, Filtered Back Projection was used and the Butterworth filter was applied with a cut-off of 0.6 and an order of 7.0. Chang's attenuation correction was applied with an attenuation coefficient of $0.12 / \mathrm{cm}$. Raw image data axial plane reconstructions of SPECT were analyzed.

All SPECT scan results were evaluated by means of the semi-quantitative total intensity index (TII). We have recently demonstrated that TII was markedly correlated with the grade of gliomas and was a reliable index in discriminating high-grade versus low-grade gliomas (20). In grade IV glioma patients, TII was used for the semi-quantitative evaluation of changes in viable glioma tissue following surgery and radiation treatment (20). In order to calculate TII, SPECT and diagnostic CT images were matched according to anatomical features observed on SPECT images including the scalp, upper parts of orbits, upper parts of frontal sinuses and the choroid plexus. Four axial slices of each CT and SPECT scan were included in the final protocol: a) at the level of the most cranial part of the choroid plexus; b) at the level of the third ventricle; c) at the level of the body of the lateral ventricles; d) at the level of the most caudal part of the choroid plexus. First, two axial slices ( $a$ and $b$ ) of the CT and SPECT images were divided into four segments each and the last two axial slices (c and d) of CT and SPECT images were divided into six segments each. Thus, each CT and SPECT scan was divided into a total of 20 segments.

The TII was calculated by evaluating the intensity of pathological ${ }^{99 \mathrm{~m}} \mathrm{Tc}-\mathrm{MIBI}$ uptake by malignant glioma tissue, separately in all 20 segments of SPECT scans, using a four-point scale according to the intensity of the tracer uptake, ranging from three (the highest intensity of visible tracer uptake) to zero (no visible tracer uptake). Specifically, 3, 2 and 1 points were assigned to the segment when the intensity of pathological ${ }^{99 \mathrm{~m}} \mathrm{Tc}-\mathrm{MIBI}$ uptake in that segment was higher, equal or lower (but more than background) than the intensity of ${ }^{99 m}$ Tc-MIBI uptake in the choroid plexus, respectively. Zero points were assigned to the segment when ${ }^{99 \mathrm{~m} T \mathrm{Tc}-M I B I}$ uptake was not evident. TII was calculated by adding the scores of all 20 segments with a possible range from 0 to 60 points.

Preoperative TII, postoperative TII and percentage change in postoperative TII when compared with preoperative TII $(\Delta$ TII) were calculated for all patients.

Statistical analysis. PASW Statistics for Windows 18.0 (IBM Corporation, Chicago, IL, USA) was used for data analyses. All continuous data are presented as the mean \pm standard deviation, and all categorical data as a number and percentage. 
Table I. Univariate analysis of the effect of prognostic factors on survival in 17 malignant glioma patients.

\begin{tabular}{|c|c|c|c|}
\hline Variable & Number of cases & Mean survival (months) & Univariate analysis P-value (log-rank) \\
\hline \multicolumn{4}{|l|}{ Gender } \\
\hline Male & 11 & $10.9 \pm 2.5$ & 0.194 \\
\hline Female & 6 & $33.5 \pm 13.3$ & \\
\hline \multicolumn{4}{|l|}{ Age (years) } \\
\hline$\geq 60$ & 10 & $14.2 \pm 6.4$ & 0.151 \\
\hline$<60$ & 7 & $25.7 \pm 9.8$ & \\
\hline \multicolumn{4}{|l|}{ Resection } \\
\hline Gross total & 11 & $19.4 \pm 5.3$ & 0.372 \\
\hline Subtotal or biopsy & 6 & $17.9 \pm 12.8$ & \\
\hline \multicolumn{4}{|l|}{ Number of symptoms } \\
\hline$\geq 4$ & 5 & $7.9 \pm 2.2$ & 0.044 \\
\hline$<4$ & 12 & $23.5 \pm 7.5$ & \\
\hline \multicolumn{4}{|l|}{ Preoperative TII } \\
\hline$\geq 12$ & 3 & $3.1 \pm 1.5$ & $<0.001$ \\
\hline$<12$ & 14 & $22.3 \pm 6.4$ & \\
\hline \multicolumn{4}{|l|}{ Postoperative TII } \\
\hline$\geq 6$ & 3 & $3.7 \pm 2.2$ & 0.001 \\
\hline$<6$ & 14 & $22.2 \pm 6.4$ & \\
\hline \multicolumn{4}{|c|}{ Decrease of $\mathrm{TII} \geq 50 \%$ after surgery } \\
\hline Yes & 12 & $24.3 \pm 7.3$ & 0.004 \\
\hline No & 5 & $6.0 \pm 2.1$ & \\
\hline
\end{tabular}

In bold, $\mathrm{p}<0.05$. TII, total intensity index.

$\mathrm{P}<0.05$ was considered to indicate a statistically significant result.

First, univariate differences in OS were tested for statistical significance using a log-rank test with respect to gender (male versus female), age ( $\geq 60$ years versus $<60$ years), extent of surgery (gross total versus subtotal or biopsy), number of preoperative symptoms ( $\geq 4$ symptoms versus $<4$ symptoms), preoperative TII ( $\geq 12$ versus $<12$ ), postoperative TII ( $\geq 6$ versus $<6)$ and $\Delta$ TII $(\geq 50 \%$ versus $<50 \%)$. Survival curves were calculated using the Kaplan-Meier method in patients with preoperative TII of $<12$ versus preoperative TII of $\geq 12$ and in patients with $\Delta$ TII of $<50 \%$ versus $\Delta$ TII of $\geq 50 \%$.

Next, a multivariate Cox regression analysis was used to analyze possible independent prognostic factors of OS. The forward-stepwise model selection procedure was used (P-value of likelihood-ratio test $<0.05$ as inclusion criterion and $>0.10$ as exclusion criterion) to define the final model. The following variables were entered to the model: gender, age at the time of surgery, number of symptoms prior to surgery, extent of resection, preoperative TII, postoperative TII and $\Delta$ TII.

Finally, patients who survived 12 months or more following surgery were compared to patients who survived less than 12 months following surgery in terms of age at the time of surgery, gender, number of symptoms prior to surgery, preoperative TII, postoperative TII and $\Delta$ TII using an independent samples t-test for continuous data and two-sided Fisher's exact test for categorical data.

\section{Results}

The mean preoperative TII was $8.8 \pm 2.6$, ranging from 4 to 12 . Following surgery, TII decreased in 16 patients and increased in 1 patient. The mean postoperative TII was $4.0 \pm 3.1$, ranging from 0 to 13 , and the mean $\Delta$ TII was $55.9 \pm 27.9 \%$, ranging from a TII increase of $8.3 \%$ to a TII decrease of $100 \%$. The mean OS time was $18.9 \pm 23.7$ months and the median OS time was 12.4 months, ranging from 1.4 to 88 months. There were nine $(45 \%)$ 1-year survivors and three $(15 \%) 2$-year survivors.

In univariate analyses using the log-rank test, we identifed that a greater number of preoperative neurological symptoms, higher preoperative TII, higher postoperative TII and lower $\Delta$ TII were associated with OS (Table I). Specifically, worse OS survival was associated with a preoperative TII of $\geq 12$ when compared to a preoperative TII of $<12$ (3.1 \pm 1.5 months versus $22.3 \pm 6.4$ months, respectively; $p<0.001$; Fig. 1 ), with a postoperative TII of $\geq 6$ when compared to a postoperative TII of $<6(3.7 \pm 2.2$ months versus $22.2 \pm 6.4$ months, respectively; $\mathrm{p}=0.001)$ and with a $\Delta$ TII of $<50 \%$ when compared to a $\Delta$ TII of $\geq 50 \%(6.0 \pm 2.1$ months versus $24.3 \pm 7.3$ months, respectively; $\mathrm{p}=0.004$; Fig. 2). Also, patients with $\geq 4$ neurological symptoms prior to surgery had a worse OS when compared to patients with $<4$ preoperative neurological symptoms $(7.9 \pm 2.2$ months versus $23.5 \pm 7.5$ months, respectively, $\mathrm{p}=0.004)$.

In multivariate analyses, postoperative TII ( $\mathrm{p}=0.008 ; 95 \%$ confidence interval, 1.12-2.13\%), number of preoperative 
Table II. Comparison of patients with overall survival of 12 months or more and less than 12 months following surgery.

\begin{tabular}{|c|c|c|c|}
\hline & \multicolumn{2}{|c|}{ Overall survival } & \multirow[b]{2}{*}{ P-value } \\
\hline & 12 months or more & Less than 12 months & \\
\hline Age, mean $\pm \mathrm{SD}$, years & $59.6 \pm 9.4$ & $65.3 \pm 6.3$ & 0.17 \\
\hline Gender, male/female & $5 / 4$ & $6 / 2$ & 0.40 \\
\hline Number of preoperative symptoms, mean \pm SD & $2.7 \pm 0.9$ & $3.1 \pm 1.0$ & 0.33 \\
\hline Resection, total/subtotal or biopsy & $8 / 9$ & $3 / 8$ & 0.05 \\
\hline Preoperative TII, mean \pm SD & $8.0 \pm 2.6$ & $9.6 \pm 2.6$ & 0.21 \\
\hline Postoperative TII, mean \pm SD & $2.3 \pm 1.7$ & $5.9 \pm 3.4$ & 0.01 \\
\hline Percentage change in TII following surgery, mean \pm SD & $70.0 \pm 21.7$ & $40.1 \pm 26.4$ & 0.02 \\
\hline
\end{tabular}

In bold, $\mathrm{p} \leq 0.05$. TII, total intensity index.

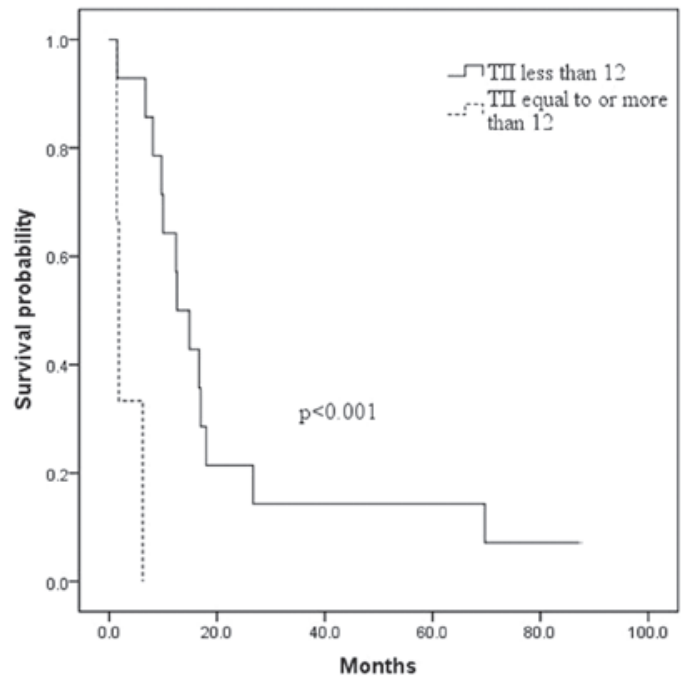

Figure 1. Kaplan-Meier estimate of overall survival for patients with a total intensity index (TII) lower than 12 points and for patients with a TII equal to or greater than 12 points.

neurological symptoms $(\mathrm{p}=0.02 ; 95 \%$ confidence interval, $1.19-5.90 \%)$ and gender $(\mathrm{p}=0.03 ; 95 \%$ confidence interval, 1.21-15.98\%) were found to be factors with independent prognostic value.

Patients who survived more than 12 months following surgery had significantly higher rates of gross total resection $(n=8 / 9(89 \%)$ versus $n=3 / 8(38 \%)$, respectively; $p=0.05)$, lower postoperative TII (2.3 \pm 1.7 versus $5.9 \pm 3.4$, respectively; $\mathrm{p}=0.01)$ and greater $\Delta \mathrm{TII}(70.0 \pm 21.7$ versus $40.1 \pm 26.4$, respectively; $\mathrm{p}=0.02$ ) when compared to patients who survived less than 12 months following surgery (Table III).

\section{Discussion}

The main finding of the present study was that a higher tracer uptake on SPECT scans was associated with a worse survival in malignant glioma patients. Specifically, we identified that higher postoperative ${ }^{99 \mathrm{~m}} \mathrm{Tc}-\mathrm{MIBI}$ uptake was an independent predictor of worse survival in malignant glioma patients. In univariate

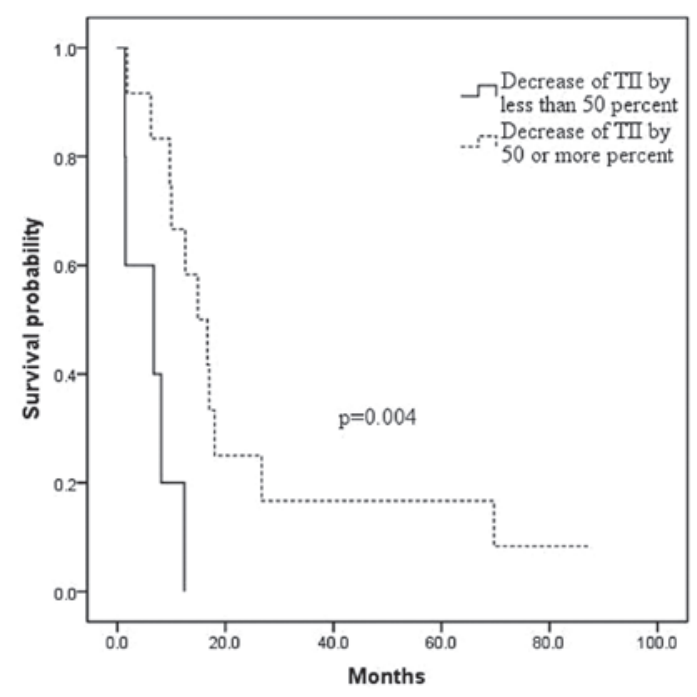

Figure 2. Kaplan-Meier estimate of overall survival for patients with a decrease in postoperative total intensity index (TII) when compared to a preoperative TII of less than $50 \%$ and of $50 \%$ or more.

analyses, worse OS was associated with higher preoperative and postoperative ${ }^{99 \mathrm{~m}} \mathrm{Tc}-\mathrm{MIBI}$ uptake as well as with a smaller decrease in ${ }^{99 m}$ Tc-MIBI uptake following surgery. Finally, patients who survived one year or more following surgery had a significantly lower postoperative ${ }^{99 \mathrm{~m}}$ Tc-MIBI uptake and a higher decrease in ${ }^{99 \mathrm{~m} T c-M I B I}$ uptake following surgery.

To the best of our knowledge, this is the first study evaluating the association of preoperative and postoperative ${ }^{99 \mathrm{~m}} \mathrm{Tc}-\mathrm{MIBI}$ uptake as well as changes in ${ }^{99 \mathrm{~m}} \mathrm{Tc}-\mathrm{MIBI}$ uptake following surgery with survival in the same cohort of malignant glioma patients. It is also the first study evaluating the association between ${ }^{99} \mathrm{~m}$ Tc-MIBI uptake assessed using the semi-quantitative TII method and survival of malignant glioma patients. The main findings were that higher ${ }^{99 \mathrm{~m}} \mathrm{Tc}-\mathrm{MIBI}$ uptake following surgery was independently associated with worse OS of malignant glioma patients after adjusting for age, gender, extent of surgery and clinical disease severity prior to surgery (number of neurological symptoms). In addition, in univariate analyses, higher ${ }^{99 \mathrm{~m} T c-M I B I}$ uptake before surgery $(\mathrm{TII} \geq 12)$, 
after surgery $(\mathrm{TII}<6)$ and a smaller decrease in ${ }^{99 \mathrm{~m}} \mathrm{Tc}-\mathrm{MIBI}$ uptake after surgery were all found to be associated with worse survival. Finally, patients who survived more than a year following surgery had significantly lower postoperative TII and a more pronounced decrease in ${ }^{99 \mathrm{~m}} \mathrm{Tc}-\mathrm{MIBI}$ uptake following surgery. Together, our findings suggest that tracer uptake by malignant glioma on SPECT scans evaluated using a semiquantitative TII method may be valuable predictors of survival in malignant glioma patients. However, our findings remain to be replicated in larger samples of malignant glioma patients.

Currently, there are a few studies evaluating the association between uptake of different tracers on SPECT scans and survival of glioma patients. In a recent study, Alexiou et al (2010) evaluated the prognostic value of preoperative ${ }^{99 \mathrm{~m}} \mathrm{Tc}$-tetrofosmin uptake in predicting the OS of 18 GBM patients and found that higher ${ }^{99 \mathrm{~m}} \mathrm{Tc}$-tetrofosmin uptake was associated with significantly worse survival (16). Another study reported that higher preoperative thalium-201 uptake was associated with significantly worse survival in high grade glioma patients (23). However, in a subgroup of patients diagnosed with WHO grade IV gliomas there were no statistically significant differences in survival between the low lesion to normal tissue ratio $(\mathrm{L} / \mathrm{N})$ ratio group and the high L/N ratio group. Beauchesne et al (2004) reported that larger metabolic tumor volume assessed using the ${ }^{99 \mathrm{~m}} \mathrm{Tc}-\mathrm{MIBI}$ at the end of radiation therapy was associated with worse prognosis of malignant glioma patients (17). Another study by the same group demonstrated that a larger tumor volume and a higher $\mathrm{L} / \mathrm{N}$ ratio were associated with worse prognosis of malignant glioma patients following treatment failure (18). To the best of our knowledge, we were the first to report on the association of a change in tracer uptake following surgery with survival of malignant glioma patients. Also, we were the first to report that higher preoperative ${ }^{99 \mathrm{~m}} \mathrm{Tc}-\mathrm{MIBI}$ uptake was correlated with worse survival. Higher uptake of fluorine-18 (18F)-2-deoxyglucose (FDG) on PET scans was reported to be a reliable predictor of worse survival in glioma patients $(24,25)$. However, the use of PET remains limited due to its high cost, in contrast to SPECT, which is a cheaper and more widely available diagnostic modality even in less developed countries.

Malignant gliomas have increased metabolic needs, increased cellular mitochondrial content and maintain the negative mitochondrial transmembrane potential that is associated with the active diffusion of ${ }^{99 \mathrm{~m}} \mathrm{Tc}-\mathrm{MIBI}$ into the mitochondria $(7,10)$. Higher ${ }^{99 \mathrm{~m}} \mathrm{Tc}-\mathrm{MIBI}$ uptake was demonstrated to correlate with biological markers of malignancy of malignant gliomas, including aneuploidy level and percentage of cells in the S-phase fraction, and with proliferative activities of glioma as assessed by the means of Ki-67 $(26,27)$. In the majority of previous studies, tracer uptake was evaluated using the $\mathrm{L} / \mathrm{N}$ ratio; the most widely used index for semi-quantitative evaluation of brain SPECT results. However, the L/N ratio corresponds to tracer uptake at the point of the brain tumor where tracer uptake is the highest, but does not include quantitative evaluation of the total tracer uptake area. TII is a semi-quantitative index that corresponds to tracer uptake in 20 segments of brain SPECT and is an index of malignancy and size of glioma. TII is a highly sensitive and specific method for identifying high-grade gliomas and may be used for the follow-up of grade IV glioma patients following surgery and radiation treatment when surrounding edema prevents accurate interpretation of CT and MRI scans (20). We have recently revealed that higher ${ }^{99 \mathrm{~m}} \mathrm{Tc}-\mathrm{MIBI}$ uptake prior to surgery evaluated using TII was positively correlated with histological WHO glioma grade $(\mathrm{r}=0.64)(20)$. Therefore, TII may be used to supplement the $\mathrm{L} / \mathrm{N}$ ratio for initial diagnosis and at follow-up.

We found that more radical resection of high-grade gliomas as evaluated by the means of SPECT was associated with improved OS. Specifically, a decrease in TII of more than $50 \%$ following surgery and a lower postoperative TII were associated with longer survival. Also, patients who survived more than a year following surgery had higher rates of total resection, lower postoperative tracer uptake and a higher decrease in TII following surgery when compared to patients who survived less than a year following surgery. Currently used multimodal treatment algorithms for malignant gliomas include maximally safe microsurgical resection followed by chemotherapy and radiotherapy. However, despite recent advances in chemotherapy and radiotherapy, maximally safe microsurgical resection remains the pivotal treatment modality of malignant glioma patients and more radical resection is associated with increased survival $(28,29)$.

We have also identifed that more preoperative neurologic symptoms were associated with worse OS, suggesting that preoperative neurological status is an important marker of disease severity and possesses an important prognostic value in malignant glioma patients. A recent study by Chaichana et al has also reported that preoperative clinical symptoms, including motor and language deficit, were independent predictors of poor survival in GBM patients undergoing resection (9).

Limitations of this study should be acknowledged. Firstly, standard chemotherapy with temozolomide was not available at the time of the study in Lithuania; therefore, the value of TII in predicting the survival of patients receiving combined radiotherapy and chemotherapy should be addressed in the future. Also, other clinical (e.g., functional status) and genetic (e.g., MGMT promoter methylation) prognostic markers were not systematically evaluated and therefore were not included in the analyses. Finally, due to the small sample size, our findings need to be considered with caution until replicated in a larger sample of malignant glioma patients. Furthermore, more advanced nuclear medicine imaging modalities, including PET and SPECT/CT were not used at our institution at the time of the study.

It should be noted that GBM patients were considered together with one gliosarcoma patient, as it was previously shown that both types of tumors share substantial clinical and genetic similarities, have similar survival rates, and should therefore be considered together in terms of treatment and enrolment to research protocols $(6,7)$.

In conclusion, the results of this study suggest that higher ${ }^{99 \mathrm{~m}} \mathrm{Tc}-\mathrm{MIBI}$ uptake before and after surgery and less pronounced decrease of ${ }^{99 \mathrm{~m}} \mathrm{Tc}-\mathrm{MIBI}$ uptake after surgery are associated with worse survival of malignant glioma patients. Evaluation of tracer uptake by means of TII during perioperative periods may be used as an additional prognostic factor of malignant glioma patients. However, further studies in larger samples are required. 


\section{References}

1. DeAngelis: LM: Brain tumors. N Engl J Med 344: 114-23, 2001

2. Schwartzbaum JA, Fisher JL, Aldape KD, Wrensch M: Epidemiology and molecular pathology of glioma. Nat Clin Pract Neurol 2: 494-503, 2006.

3. Liu Y, Shete S, Etzel CJ, Scheurer M, Alexiou G, Armstrong G, et al: Polymorphisms of LIG4, BTBD2, HMGA2, and RTEL1 genes involved in the double-strand break repair pathway predict glioblastoma survival. J Clin Oncol 28: 2467-2474, 2010.

4. Scott JN, Rewcastle NB, Brasher PM, Fulton D, MacKinnon JA, Hamilton $\mathrm{M}$, et al: Which glioblastoma multiforme patient will become a long-term survivor? A population-based study. Ann Neurol 46: 183-188, 1999.

5. Stupp R, Mason WP, van den Bent MJ, Weller M, Fisher B, Taphoorn MJ, et al: Radiotherapy plus concomitant and adjuvant temozolomide for glioblastoma. N Engl J Med 352: 987-996, 2005.

6. Galanis E, Buckner JC, Dinapoli RP, Scheithauer BW, Jenkins RB, Wang $\mathrm{CH}$, et al: Clinical outcome of gliosarcoma compared with glioblastoma multiforme: North Central Cancer Treatment Group results. J Neurosurg 89: 425-430, 1998.

7. Lutterbach J, Guttenberger R, Pagenstecher A: Gliosarcoma: a clinical study. Radiother Oncol 61: 57-64, 2001.

8. Adamson C, Kanu OO, Mehta AI, Di C, Lin N, Mattox AK, et al: Glioblastoma multiforme: a review of where we have been and where we are going. Expert Opin Investig Drugs 18: 1061-1083, 2009.

9. Chaichana K, Parker S, Olivi A and Quiñones-Hinojosa A: A proposed classification system that projects outcomes based on preoperative variables for adult patients with glioblastoma multiforme. J Neurosurg 112: 997-1004, 2010.

10. El Hindy N, Bachmann HS, Lambertz N, Adamzik M, Nücke $\mathrm{H}$, Worm $\mathrm{K}$, et al: Association of the $\mathrm{CC}$ genotype of the regulatory BCL2 promoter polymorphism (-938C $>$ A) with better 2 -year survival in patients with glioblastoma multiforme. J Neurosurg 114: 1631-1639, 2011.

11. Cheng X, Li Y, Xu Z, Li D and Wang J: A meta-analysis of (99m) Tc-MIBI SPECT for detection of recurrent glioma after radiation therapy. J Clin Neurosci 18:307-312, 2011.

12. Kallén K, Burtscher IM, Holtås S, Ryding E and Rosén I: 201Thallium SPECT and 1H-MRS compared with MRI in chemotherapy monitoring of high-grade malignant astrocytomas. J Neurooncol 46: 173-185, 2000.

13. Prigent-Le Jeune F, Dubois F, Perez S, Blond S and Steinling M: Technetium-99m sestamibi brain SPECT in the follow-up of glioma for evaluation of response to chemotherapy: first results. Eur J Nucl Med Mol Imaging 31: 714-719, 2004.

14. Schwartz RB, Holman BL, Polak JF, Garada BM, Schwartz MS, Folkerth R, et al: Dual-isotope single-photon emission computerized tomography scanning in patients with glioblastoma multiforme: association with patient survival and histopathological characteristics of tumor after high-dose radiotherapy. J Neurosurg 89: 60-68, 1998.

15. Ak I, Gulbas Z, Altinel F and Vardareli E: Tc-99m MIBI uptake and its relation to the proliferative potential of brain tumors. Clin Nucl Med 28: 29-33, 2003.
16. Alexiou GA, Tsiouris S, Kyritsis AP, Fotakopoulos G, Goussia A, Voulgaris S, et al: The value of $99 \mathrm{mTc}$-tetrofosmin brain SPECT in predicting survival in patients with glioblastoma multiforme. J Nucl Med 51: 1923-1926, 2010.

17. Beauchesne P, Pedeux R, Boniol M and Soler C: $99 \mathrm{mTc}$-sestamibi brain SPECT after chemoradiotherapy is prognostic of survival in patients with high-grade glioma. J Nucl Med 45: 409-413, 2004.

18. Beauchesne P and Soler C: Correlation of $99 \mathrm{mTc}-\mathrm{MIBI}$ brain spect (functional index ratios) and survival after treatment failure in malignant glioma patients. Anticancer Res 22: 3081-3085, 2002.

19. Nagamachi S, Jinnouchi S, Nabeshima K, Nishii R, Flores L II, Kodama T, et al: The correlation between $99 \mathrm{mTc}-\mathrm{MIBI}$ uptake and MIB-1 as a nuclear proliferation marker in glioma - a comparative study with 201Tl. Neuroradiology 43: 1023-1030, 2001.

20. Deltuva VP, Jurkiene N, Kulakiene I, Bunevicius A, Matukevicius A and Tamasauskas A: Introduction of novel semiquantitative evaluation of $99 \mathrm{mTc}-\mathrm{MIBI}$ SPECT before and after treatment for glioma. Medicina (Kaunas) 48 (1): 15-21, 2012.

21. Filippi L, Santoni R, Manni C, Danieli R, Floris R and Schillaci O: Imaging primary bain tumors by single-photon emission computerized tomography (SPECT) with technetium$99 \mathrm{~m}$ sestabimi (MIBI) and tetrofosmin. Current Medical Imaging Reviews 1: 61-66, 2005.

22. Henkin RE, Bova D, Dillehay GL, Halama JR, Karesh SM, Wagner RH, et al (eds): Nuclear Medicine. 2nd edition. Mosby Elsevier, Philadelphia, pp137-153, 2006.

23. Semba T, Sugawara Y, Ochi T, Fujii T, Mochizuki T and Ohnishi T: Thallium-201 SPECT in prognostic assessment of malignant gliomas treated with postoperative radiotherapy. Ann Nucl Med 20: 287-294, 2006.

24. Padma MV, Said S, Jacobs M, Hwang DR, Dunigan K, Satter M, et al: Prediction of pathology and survival by FDG PET in gliomas. J Neurooncol 64: 227-237, 2003.

25. Patronas NJ, Di Chiro G, Kufta C, Bairamian D, Kornblith PL, Simon R, et al: Prediction of survival in glioma patients by means of positron emission tomography. J Neurosurg 62: 816-822, 1985.

26. Chen WS, Luker KE, Dahlheimer JL, Pica CM, Luker GD and Piwnica-Worms D: Effects of MDR1 and MDR3 P-glycoproteins, MRP1, and BCRP/MXR/ABCP on the transport of $(99 \mathrm{~m})$ Tc-tetrofosmin. Biochem Pharmacol 60: 413-426, 2000.

27. Delmon-Moingeon LI, Piwnica-Worms D, Van den Abbeele AD, Holman BL, Davison A and Jones AG: Uptake of the cation hexakis(2-methoxyisobutylisonitrile)-technetium-99m by human carcinoma cell lines in vitro. Cancer Res 50: 2198-202, 1990.

28. Pichlmeier U, Bink A, Schackert G, Stummer W; ALA Glioma Study Group: Resection and survival in glioblastoma multiforme: an RTOG recursive partitioning analysis of ALA study patients. Neuro Oncol 10: 1025-1034, 2008.

29. Sanai N, Polley MY, McDermott MW, Parsa AT and Berger MS: An extent of resection threshold for newly diagnosed glioblastomas. J Neurosurg 115: 3-8, 2011.. 